COASTAL ENGINEERING 2010

\title{
HYDRODYNAMIC PERFORMANCE OF A FREE SURFACE SEMICIRCULAR PERFORATED BREAKWATER
}

\author{
Hee Min Teh', Vengatesan Venugopal ${ }^{2}$, Tom Bruce ${ }^{3}$
}

\begin{abstract}
The increasing importance of the sustainability challenge in coastal engineering has led to the development of free surface breakwaters of various configurations. In this study, the hydrodynamic characteristics of a perforated free surface, semicircular breakwater (SCB) are investigated for irregular wave conditions. The hydrodynamic performance of the breakwater is evaluated in the form of transmission, reflection and energy dissipation coefficients, which are then presented as a function of the relative immersion depth $(D / d)$ and the relative breakwater width $\left(B / L_{p}\right)$, where $D=$ the depth of immersion, $d=$ the water depth, $B=$ the breakwater width and $L_{p}=$ the wavelength corresponding to the peak wave period. It is found that the wave attenuation ability of the SCB model improves with the increase of $D / d$ and $B / L_{p}$. The SCB performs better as an energy dissipater than as a wave reflector. Based on the analysis of measured data, some empirical equations are proposed to predict the performance of the breakwater under varying immersion depths. The behaviour of wave transformation around and within the breakwater's chamber is discussed. Also, the measured horizontal wave forces acting on the SCB are reported.
\end{abstract}

Keywords: semicircular breakwater; wave transmission; wave reflection; energy dissipation; wave force

\section{INTRODUCTION}

Bottom-seated breakwaters are traditionally built to provide sea defence and coastal protection. These structures are generally massive in size, associated with large scales in construction materials, effort and cost. The development of large breakwater schemes may trigger some effects on neighbouring coastal environment, e.g. large amount of wave reflection. Waves reflected from the breakwater may pose navigational hazard to small vessels, toe's scour and even explosive clapotis in front of the structure.

In the $21^{\text {st }}$ century, the increasing importance of the sustainability challenge in coastal engineering has led to the research effort in developing various 'soft' measures to provide other alternatives to the conventional breakwaters, including free surface breakwaters. Free surface breakwaters, sometimes also termed as open-type breakwaters, are essentially barriers located near the water surface where the energy flux is maximal. The total height of such barriers is usually far smaller than the water depth. They reduce wave energy by wave reflection and/or dissipation. The barriers can be fixed by a group of piles or held floating by different mooring systems. Free surface breakwaters have been found to be advantageous for the development of some facilities where a certain amount of wave transmission into the sheltered area is acceptable, or in cases where incident wave climates are not severe.

On the whole, the majority of the free surface breakwaters reduce the energy transmitted to the leeside of the structures by wave reflection. In many cases, the effect of wave reflection in front of the breakwater is regarded as a potential risk to the sea navigation. The present investigation is motivated by the need to develop a free surface breakwater that could meet functional, economic and safety requirements. Although several experimental and theoretical studies have been reported in the literature on the performance characteristics of cylindrical and quadrant front face free surface breakwaters, to the knowledge of the authors, the performance of the perforated semicircular breakwater (which could be supported on piles or jacket structures) located near the free surface has not yet been investigated. In the present work, a model of a perforated semicircular free surface breakwater (SCB) has been constructed and tested under various wave conditions.

The present study aims to evaluate the hydrodynamic performance of a perforated SCB through a series of systematic physical model tests. It is essential to understand how the SCB interacts with the wave system to produce desirable performance characteristics, which then would aid with the optimisation process of the breakwater design. As a result of the data analysis, several robust prediction formulae are suggested to determine the hydrodynamic performance of the breakwater within the test limit.

\footnotetext{
${ }^{1}$ Postgraduate student, ${ }^{2}$ Lecturer, ${ }^{3}$ Senior lecturer

Institute for Energy Systems, School of Engineering, The University of Edinburgh, Faraday Building,

The King's Buildings, Mayfield Road, Edinburgh, EH9 3JL, UK.
} 


\section{LITERATURE REVIEW}

In the past, free surface breakwaters with various inventive designs have been proposed and tested by researchers. Some are designed to withstand the wave energy by their own weights; and the others suppress the wave energy by means of unique structural features that are cost-and-space effective. Breakwater designs, that are both economically and functionally viable, are somehow difficult to achieve in reality. In general, free surface breakwaters can be classified as (a) solid-type; (b) plate-type; (c) caisson-type; and (d) multipart-type. Examples of each type of free surface breakwater are shown in Figure 1. The literature on some of the free surface breakwaters are highlighted in the following sections and these are limited only to fixed free surface breakwaters.

\section{(a) Solid-type}

Solid-type barriers are relatively bulky than other types of free surface breakwater. The box-type breakwater has the simplest form of design in the development of free surface breakwaters. Koutandos \& Prinos (2005) provided the design formulae for wave transmission, reflection and dissipation, respectively, for a box-type breakwater. The experimental results showed that the box-type breakwater was a highly reflective structure, particularly when the breakwater was immersed at larger draft. It achieved almost $35 \%$ wave attenuation with a resultant reflection of $80 \%$ of the incident wave energy when the breakwater width was $30 \%$ of the incident wavelength, and the breakwater draft was $20 \%$ to $33 \%$ of the total water depth. In another work, Koutandos (2007) numerically studied the hydrodynamic performance of two partially immersed box type breakwaters separated by a distance. The magnitudes of flow velocities, turbulence kinetic energy and vortices observed were much higher in the region of the first barrier than those in the second one.

Li et al. (2005) modelled the characteristics of wave transmission past an infinitely long cylinder in shallow, transitional and deep waters using the modified Tsay \& Liu's (1983) approximation. The numerical results showed decrement in wave transmission with the increase in breakwater size and draft. Sundar \& Sabbarao (2003) developed a solid quadrant front face pile-supported breakwater, which was designed to dissipate the excessive wave energy by reflection from the quadrant front face on its top portion during high tides and to dissipate the wave energy with its closely spaced piles at its bottom during low tides. Their regular wave test results showed a radical amplification of wave reflection by more than $50 \%$ when the breakwater radius is above $23 \%$ of the wavelength.

Trapezoidal barrier is another solid-type free surface structure that may be considered in the design of breakwaters. Koftis \& Prinos (2005) commented that the trapezoidal barrier was more efficient than the box-type barrier in both wave attenuation and reduction in wave reflection. The trapezoidal structures trigger more wave-structure hydrodynamics, including vortices produced at the edges of the structure, the associated turbulence, wave run-up and run-down on the sloping face of the structure.

\section{(b) Plate-type}

Extensive experimental studies have been conducted on a fixed horizontal plate barrier consisting of a single-, a twin- and multiple plates located at different submergence depths. Hu et al. (2002) noted that wave transmission had a positive relation with the vertical distance between the water surface and the submerged horizontal plate. Patarapanich \& Cheong (1989) found that wave reflection induced by the twin plate system was the primary cause of wave height reduction. Neelamani \& Gayathri (2006) suggested the optimum wave reflection occurred when the spacing of the twin plates was $40 \%$ of the water depth. Neelamani \& Rajendran (2002a \& 2002b) experimentally studied the performance characteristics of the $T$-type and $\perp$-type breakwaters. They deduced that T-type breakwater was superior to the $\perp$-type breakwater by about $20-30 \%$ under identical testing conditions. Neelamani \& Vedagiri (2002) investigated the geometrical effect of the partially immersed $H$-shape barrier. The experimental results indicated that the downward extension of rear wall potentially reduced the wave action at its front wall.

\section{(c) Caisson-type}

The development of free surface, caisson-type breakwaters are essentially initiated by the conventional caisson breakwater seated on the rubble mound. These rectangular structures are generally smaller in size and have impermeable walls around the water boundary. To reduce wave reflection in front of the structure, Brossard et al. (2003) suggested adding an absorbing porous caisson at the seaside of the $\sqcup$-type structure. They found that the great efficiency of such caisson on wave transmission characteristics was accompanied by a larger transfer of energy from the fundamental 
mode to the higher harmonics through non-linear processes due to the formation of strong vortices at the orifices of the perforated wall. An increase of the immersion depth of the absorbing $\sqcup$-type breakwater was more efficient than an increase of the width of the structure in attenuating the wave energy.

\begin{tabular}{|c|c|c|}
\hline Types of breakwater & Features \& cross sections & References \\
\hline Solid-type & $\begin{array}{l}\text { Box } \\
\text { Cylinder } \\
\text { Quadrant front face } \\
\text { Trapezoid }\end{array}$ & $\begin{array}{l}\text { Koutandos \& Prinos (2005) } \\
\text { Koutandos (2007) } \\
\text { Li et al. (2005) } \\
\text { Sundar \& Subbarao (2003) } \\
\text { Kofis \& Prinos (2005) }\end{array}$ \\
\hline Plate-type & $\begin{array}{l}\text { Horizontal plate } \\
\text { Twin-plate } \\
\text { T-type } \\
\perp \text {-type } \\
H \text { - type }\end{array}$ & $\begin{array}{l}\text { Hu et al. (2002) } \\
\text { Usha \& Gayathri (2005) } \\
\text { Neelamani \& Gayathri (2006) } \\
\text { Neelamani \& Rajendran (2002a) } \\
\text { Neelamani \& Rajendran (2002b) } \\
\text { Neelamani \& Vegariri (2002) }\end{array}$ \\
\hline Caisson-type & $\begin{array}{l}\sqcup-\text { type } \\
\square-\text { type }\end{array}$ & $\begin{array}{l}\text { Brossard et al. (2003) } \\
\text { Günaydın \& Kebdaşlı (2004) } \\
\text { Günaydin \& Kebdaşlı (2006) } \\
\text { Koftis \& Prinos (2005) }\end{array}$ \\
\hline Multipart-type & $\begin{array}{ll}\text { Plate-type } & \\
\text { Interlaced-type } & \end{array}$ & $\begin{array}{l}\text { Wang et al. (2006) } \\
\text { Hsiao et al. (2008) }\end{array}$ \\
\hline
\end{tabular}

Figure 1. Types of free surface breakwater. 
Günaydın \& Kebdaşlı (2004) investigated the performance of solid and perforated $\sqcup$-type breakwaters, in which the vertical front wall and horizontal plate were made perforated by circular holes. Similar study was conducted for the $\sqcap$-type breakwaters with identical structure's porosity and testing conditions (Günaydın \& Kebdaşlı 2007). Comparing the wave attenuation performance of the $\sqcup$-type and $\sqcap$-type breakwaters, the latter was found slightly better than the former. Koftis \& Prinos (2005) numerically examined the effect of structure's shape on the hydrodynamic efficiency of free surface breakwaters using the Reynolds Averaged Navier Stokes equations; and the results showed that the wave dampening ability of the solid-type breakwaters was generally higher than the impervious $\square$ type breakwater.

\section{(d) Multipart-type}

Multipart-type breakwaters typically consist of a number of constituents interconnected and assembled by some connecting elements. The reflectivity and horizontal wave forces acting on these structures are generally weak. Wang et al. (2006) developed such a breakwater composed of several horizontal plates aiming to retard the fluid particle motions in the upright direction. This breakwater brought down the height of incident waves by half when the width of the structure was about $25 \%$ of the wavelength. Wave transmission increased with increased gaps between the horizontal plates; however, the influence of relative gap to wave reflection was reported to be insignificant. Hsio et al. (2008) studied the wave energy dissipation due to the porous-pile structure. The structure consisted of multiple arrays of 'pile-like' elements interconnected with each other. The maximum reflected wave height-to-incident wave height ratios (reflection coefficients) reported for structures with porosities of $40 \%, 60 \%$ and $80 \%$ were $0.6,0.5$ and 0.3 , respectively.

The above literature indicates that different types of breakwaters could produce different hydrodynamic performance which entirely depends on structure's features and testing conditions. It is hoped that the perforated SCB considered in the present research would produce performance characteristics which could be comparable to some of the above illustrated breakwater designs.

\section{EXPERIMENTAL SETUP}

\section{Semicircular model}

The present studies deal with surface waves in which the scaling is in accordance with the Froude scaling law. The law assumes that gravity is the dominant physical force balancing the initial force, and the effects of other forces are negligible. These assumptions may lead to scale effects if other forces are dominant in the problem. In the present study, the scale effects were anticipated to be minor for such a perforated structure because the tests were mostly conducted in non-breaking wave conditions and the flow Reynolds numbers in the breakwater's chamber were always in fully-turbulent flow range $(186764<R e<349404)$. The structure permits a significant portion of wave to enter the structure, thus the viscous scale effect is usually not a problem in the model (Hughes 1993).

The 1:20 scaled SCB model, as shown in Figure 2, was constructed from a semi-cylindrical PVC tube with a shell thickness of $10 \mathrm{~mm}$. The radius of the SCB is $0.25 \mathrm{~m}$. The breakwater length perpendicular to the wave direction is $0.395 \mathrm{~m}$. Rectangular openings are provided at the front and rear curved walls at different sizes and distributions. The dimensions of the front and rear wall openings are $10 \mathrm{~mm} \times 60 \mathrm{~mm}$ and $30 \mathrm{~mm} \times 60 \mathrm{~mm}$, respectively. The front curved wall has 6 rows by 4 columns of openings spread out across the quadrant surface area, giving a porosity of $9 \%$. The perforation of the front wall is created to promote energy dissipation as water flows through the openings. The rear wall openings extend from the crest of the breakwater with a matrix of $2 \times 4$. The wall below the rear openings is solid. The rear openings are designed to reduce the volume of the overtopping waves and to provide a getaway to the excessive run up at the rear wall. Two clear Perspex sheets cut out into the shape of semicircles were attached at both ends of the model to increase its stability against wobbling during wave impact and to provide visibility to the wave interaction taking place in the breakwater's chamber. 

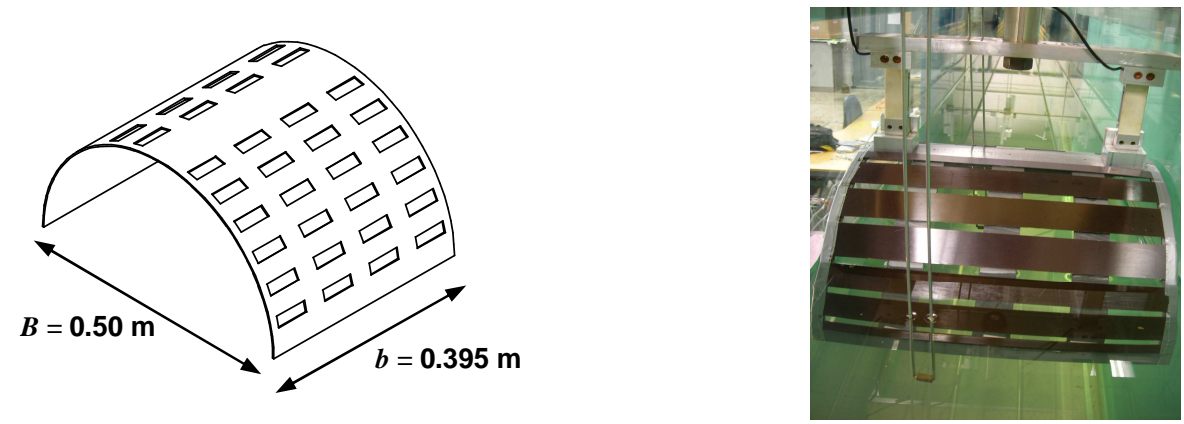

Figure 2. SCB model; Isometric view (left) and front view (right).

\section{Instrumentation}

The laboratory tests were conducted in a $22 \mathrm{~m}$ long, $0.4 \mathrm{~m}$ wide and $0.7 \mathrm{~m}$ deep wave flume in the Hydraulics Laboratory of the School of Engineering, The University of Edinburgh. The flap-type, active absorption wave generator was used to produce series of random waves. At the down-wave end of the flume, passive wave absorbing "beaches" were installed to minimise the reflection of incident waves from the end wall of the wave flume during the experiments. The beach was capable of absorbing waves more than $95 \%$ in the flume. The test section was located at a distance of $12 \mathrm{~m}$ from the wave generator. Six conductance type wave probes (WP1, WP2, WP3, WP4, WP5 and WP6) were used to record the water surface elevation at different locations in the flume. Three wave probes (WP1, WP2 and WP3) located toward the seaside of the model were used to separate the incident and reflected waves using Mansard \& Funke Method (1980). WP3 was located at a minimum distance of half of the longest wave length generated in the flume. The separation distances between the three probes were altered for each wave peak period. The probe WP4 located at a distance of $50 \mathrm{~mm}$ from the seaward wall of the model was used to measure the surface elevation just in front of the model. Another probe WP5 was positioned through one of the rectangular openings to measure the fluctuation of water level within the breakwater's chamber. The transmitted waves were measured by the probe WP6, which was located at a distance $2.5 \mathrm{~m}$ from the leeward wall of the model. The horizontal wave forces acting on the SCB model were measured by two load cells (LC1 and LC2) placed at the crest of the model. Both wave probes and load cells were carefully calibrated before each set of experiments. The present laboratory tests were conducted under controlled conditions. A schematic diagram of the experimental setup is shown in Figure 3.

\section{Testing conditions}

The waves were generated using JONSWAP spectrum in the flume. Under irregular wave condition, the test model was subjected to 11 peak periods, ranging from $0.8 \mathrm{~s}$ to $1.8 \mathrm{~s}$ in steps of $0.1 \mathrm{~s}$ at a water depth of $0.7 \mathrm{~m}$. For each peak period, at least four different significant wave heights, $H_{m 0}$, ranging from $0.04 \mathrm{~m}$ to $0.14 \mathrm{~m}$, were considered. This yielded a range of wave steepness, $H_{m 0} / L_{p}$ varying from 0.01 to 0.10 (where $L_{p}$ is the wavelength, calculated taking into the account of water depth and corresponding peak wave period). Three relative depths of immersion were attempted in this experimental study, i.e. the bottom surface of the model was lowered by $0.05 \mathrm{~m}, 0.10 \mathrm{~m}$, and $0.15 \mathrm{~m}$ relative to the still water level, such that the ratio $D / d=0.071,0.143$ and 0.214 , where $D$ and $d$ are the draft and water depth, respectively. The test environments encompassed both deep and transitional water conditions. A total of 205 test runs were conducted in this study. 

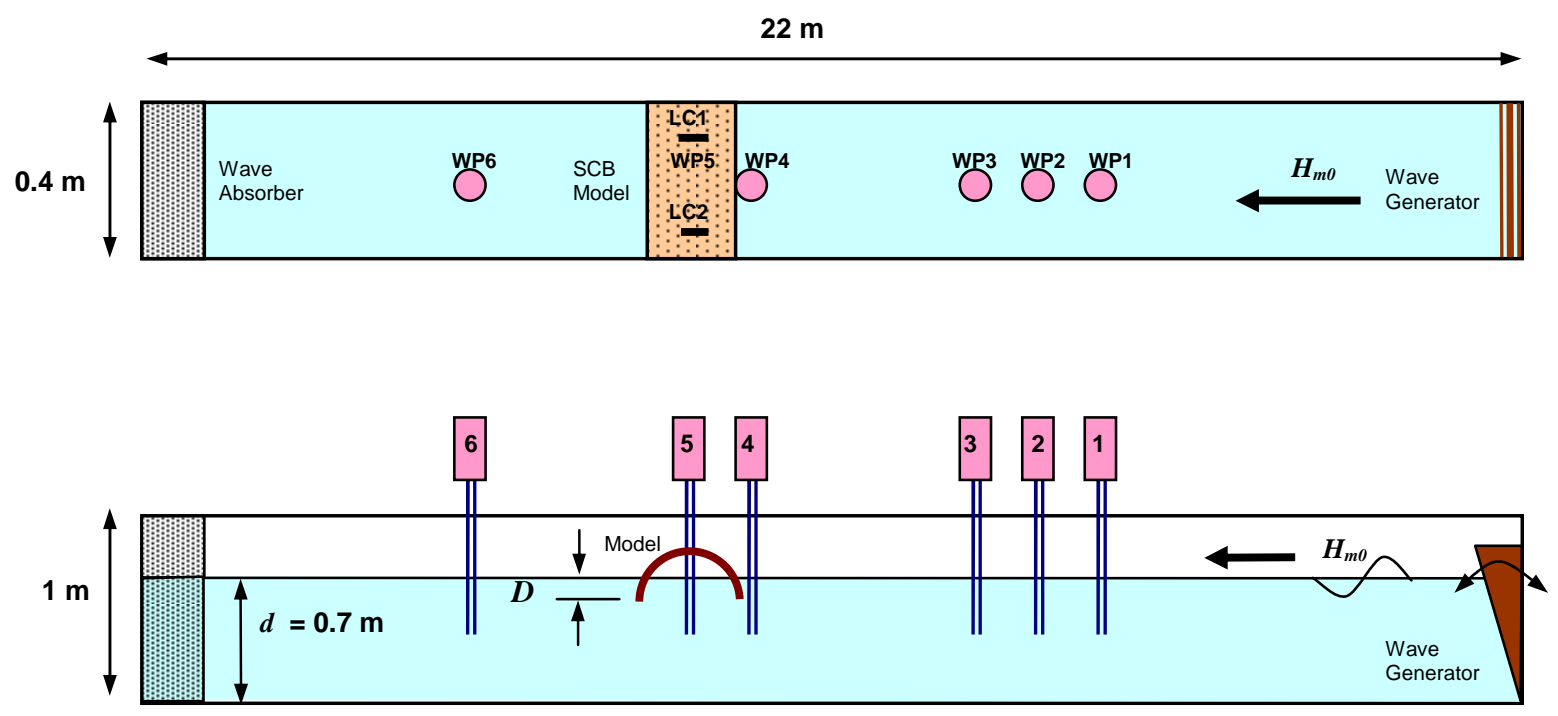

Figure 3. Experimental set-up; Plan view (top) and cross-sectional view (bottom) of the wave flume.

\section{EXPERIMENTAL RESULTS}

\section{Performance Evaluation Criteria}

The functionality of a breakwater is generally assessed by the resulting transmission coefficient $C_{T}$, reflection coefficient $C_{R}$ and dissipation coefficient $C_{L}$ as follows:

$$
\begin{gathered}
C_{T}=\frac{H_{m 0, t}}{H_{m 0}} \\
C_{R}=\frac{H_{m 0, r}}{H_{m 0}} \\
C_{L}=\sqrt{1-C_{R}^{2}-C_{T}^{2}}
\end{gathered}
$$

where $H_{m 0}$ is the significant incident wave height, and $H_{m 0, t}$ and $H_{m 0, r}$ are the significant transmitted and reflected waves, respectively. Due to difficulty in measuring energy loss, the amount of energy dissipation at the breakwater is, therefore, estimated by the law of conservation of energy, yielding Equation (3).

Wave transformations around the SCB are represented by modification coefficients. The wave characteristics in front of the breakwater is quantified by $C_{F}$

$$
C_{F}=\frac{H_{m 0, f}}{H_{m 0}}
$$

and the wave characteristics in the breakwater chamber is quantified by $C_{C}$

$$
C_{C}=\frac{H_{m 0, c}}{H_{m 0}}
$$

where $H_{m 0, f}$ and $H_{m 0, c}$ are the significant wave heights at the front and within the breakwater chamber, respectively. 


\section{Hydraulic Performance}

\section{Energy Coefficients}

The hydraulic behaviour of the SCB in random waves is investigated with respect to structure geometry, relative depth of immersion, and wave conditions. In this study, the geometrical effect of structure is represented by the relative breakwater width, $B / L_{p}$, where $B$ the diameter of the breakwater. Figure 4 shows the effect of $B / L_{p}$ on $C_{T}, C_{R}$ and $C_{L}$ for three relative depths of immersion, i.e. $D / d=$ $0.071,0.143$ and 0.214 . It is clear from Figure 4(a) that $C_{T}$ decreases with the increase in $B / L_{p}$ and $D / d$. This implies that wave attenuation of the SCB improves with the decreasing wave period and the increasing breakwater draft. The approximate reduction rates of $C_{T}$ for a unit increase of one-tenth of $B / L_{p}$ are $0.07,0.12$ and 0.16 , for $D / d=0.071,0.143$ and 0.214 , respectively. These numbers indicate a faster reduction of $C_{T}$ for higher $D / d$. Improvement of wave attenuation of the SCB9 model at higher range of $B / L_{p}$ is mainly due to energy dissipation and small amount of wave reflection, as can be seen in Figure 4(b) and 4(c).

Figure $4(\mathrm{~b})$ shows the reflective characteristics of the SCB model with respect to $B / L_{p}$. The dependence of $C_{R}$ upon $B / L_{p}$ is found to be less significant compared to that of $C_{T}$. The data points of $C_{R}$ for $D / d=0.071,0.143$ and 0.214 display mild fluctuating behaviours with $B / L_{p}$, varying from $0.10-$ $0.30,0.15-0.50$ and $0.20-0.46$, respectively. The $C_{R}$ for the respective $D / d$ seem to attain the first peak at $B / L_{p} \approx 0.25$, and subsequently diminishes slightly at $0.25<B / L_{p}<0.40$ before rising again at $0.40<B / L_{p}<0.70$. A common trend is also observed in the quadrant front face breakwater tested in regular waves (Sundar \& Subbarao 2003). In addition, the $C_{R}$ of the model is found to increase with the increasing $D / d$, particularly at $0.10<B / L_{p}<0.35$. The $C_{R}$ of $D / d=0.214$ seems to be comparable to those of $D / d=0.143$ for $0.35<B / L_{p}<0.70$. The reduction of $C_{R}$ of $D / d=0.214$ at higher range of $B / L_{p}$ could be due to (i) the occurrence of wave overtopping that limits the amount of reflected wave energy; and (ii) significant energy loss resulted from excessive wave-structure interactions at the SCB model.

Figure 4(c) displayed the energy characteristics of the SCB model for the respective $D / d$ tested. The model demonstrates its high efficiency in dissipating wave energy of the shorter period waves for all immersion depths. The larger the breakwater draft, the greater the $C_{L}$ will be resulted. At $0.1<B / L_{p}$ $<0.4$, the increment rate of $C_{L}$ for a unit increase of one-tenth of $B / L_{p}$ is approximately 0.14 for all the tested $D / d$ values. For $0.4<B / L_{p}<0.7$, the SCB models for $D / d=0.071,0.143$ and 0.214 seem to reach maximum $C_{L}$ of $0.75,0.85$ and 0.90 , respectively. Energy dissipation at this $B / L_{p}$ range can only be further enhanced by the increase of breakwater draft.

The mechanisms of energy dissipation exhibited in the SCB model were observed with the aid of digital camera and video camera. Figure 5 and 6 compare the wave-structure interaction in regular waves of period $1.3 \mathrm{~s}$ in immersion depths of $0.05 \mathrm{~m}$ and $0.15 \mathrm{~m}$, respectively. It is noted from the figures that the incident waves approached the SCB model from the right-hand side. It can be seen that the energy dissipation performance of the SCB improves with the increase in breakwater draft and the increase in wave height. As wave period increases, the SCB model becomes less dissipative as can be seen in Figure 7. From the observations during the experiments, the hydraulic processes deemed to contribute to the energy dissipation include: (i) exchange of water jet flow around the porous front wall during the passage of waves; (ii) wave run-up at the front wall; (iii) water infiltration to the breakwater's chamber; (iv) formation of eddies around the bottom walls; (v) turbulent flow within the chamber; and (v) flow instability close to the free surface at the leeside of the breakwater due to pressure difference. The number and intensity of aforementioned processes taking place at the SCB model vary with test conditions.

From Figure 4, it is also indirectly shown in the plots that the dependence of the energy coefficients upon the wave steepness is comparatively weak. Hence, the influence of wave steepness to $C_{T}, C_{R}$ and $C_{L}$ has not been taken into consideration in the empirical analysis. 
(a) Wave Transmission

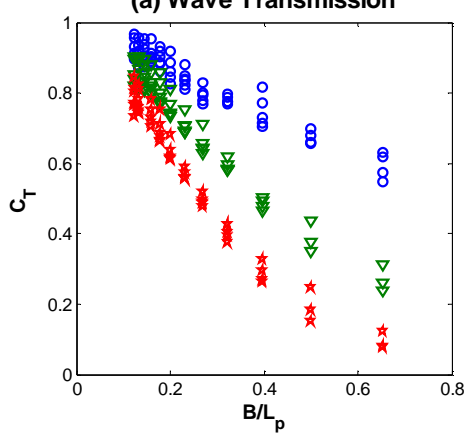

(b) Wave Reflection

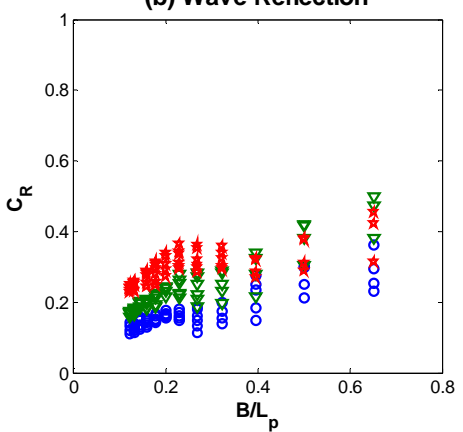

(c) Energy Dissipation

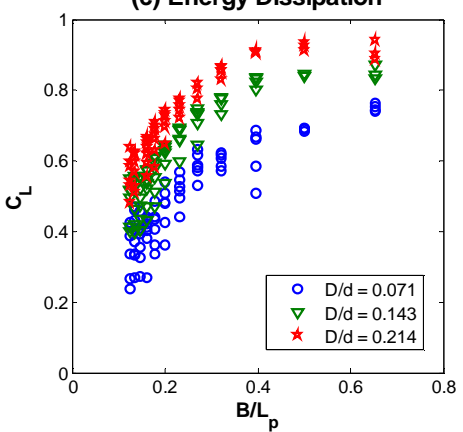

Figure 4. $C_{T}, C_{R}$ and $C_{L}$ of the SCB model.

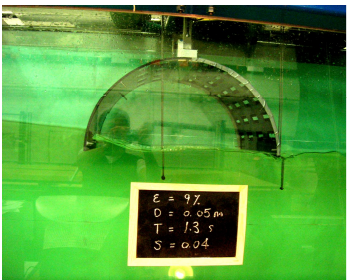

(a) $t / T=0.13$

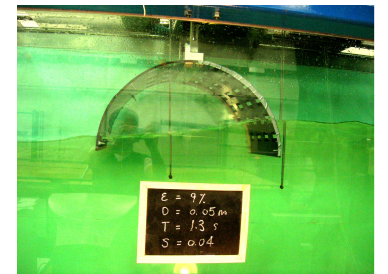

(b) $t / T=0.25$

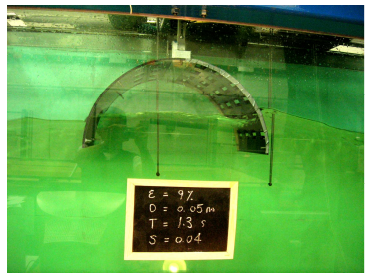

(c) $t / T=0.50$

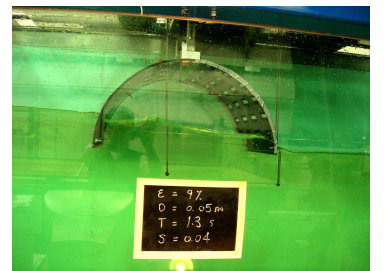

(d) $t / T=0.63$

Figure 5. Wave-structure interactions $\left(T=1.3 \mathrm{~s}, D=0.05 \mathrm{~m}, H_{i}=0.10\right)$.

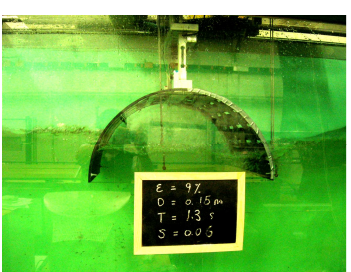

(a) $t / T=0.13$

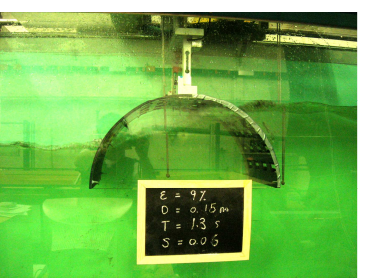

(b) $t / T=0.25$

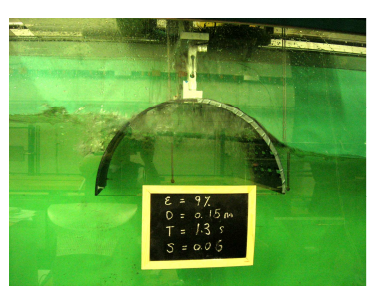

(c) $t / T=0.50$

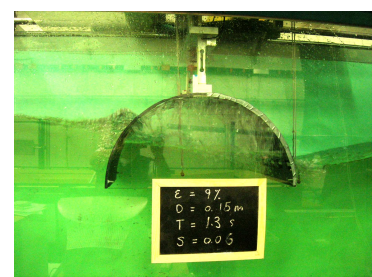

(d) $t / T=0.63$

Figure 6. Wave-structure interactions $\left(T=1.3 \mathrm{~s}, D=0.15 \mathrm{~m}, H_{i}=0.15\right)$.

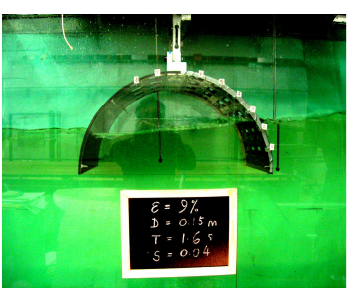

(a) $t / T=0.13$

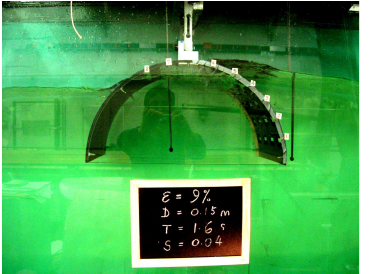

(b) $t / T=0.25$

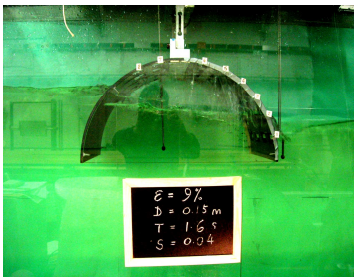

(c) $t / T=0.50$

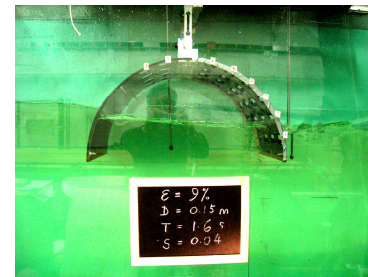

(d) $t / T=0.63$

Figure 7. Wave-structure interactions $\left(T=1.6 \mathrm{~s}, D=0.15 \mathrm{~m}, H_{i}=0.10\right)$. 


\section{Result Comparison}

It may be helpful to compare the present experimental results with those of the box-type breakwater (Koutandos \& Prinos 2005), the quadrant front face pile supported breakwater (Sundar \& Subarrao 2003), and a cylindrical structure ( $\mathrm{Li}$ et al. 2005), as presented in Figure 8. Prior to that, it is worthwhile to note some of the testing conditions of these breakwater models. The solid box-type breakwater was positioned close to the free water surface without any supporting elements beneath the structure. Whereas, the quadrant front face breakwater was supported by a group of cylindrical piles arranged in a way that the pile gap was five times greater than the diameter of the pile. Both box-type and quadrant front face breakwater models were tested in irregular waves at different depths of immersion in unidirectional waves. For the cylindrical fixed floating structure, the transmission coefficients in regular wave conditions were calculated by using the modified Tsay \& Liu's (1983) approximation. For the present comparison, $D / d \approx 0.2$ was selected for the box type breakwater and the $\mathrm{SCB}$. For the quadrant front face breakwater and the cylindrical structure, $D / d=0.31$ and 0.25 were respectively chosen as these were the minimum relative immersion depths tested by the authors. A superimpose of the data points of the respective free surface breakwater models were presented in Figure 8.

In general, the plots of the SCB and quadrant front face breakwaters exhibit similar trend with the increase in $B / L_{p}$ due to geometric similarity at the front curved walls of both models. The variation in the results is mainly due to the influence of the breakwater's porosity and immersion depth, as well as the effect of the closely spaced piles for the quadrant front face breakwater. For box type breakwater, it is seen from Figure 8(a) that the $C_{T}$ values relate well with those of the SCB and the quadrant front face breakwater. It implies that wave transmission ability is less influenced by the geometric characteristics of these free-surface breakwaters for $B / L_{p}<0.3$. The floating cylinder generally produces comparatively smaller $C_{T}$ than the rest of the breakwaters because the results were simulated in regular wave condition. In comparison to SCB and quadrant types, the box-type breakwater exhibits much higher reflective characteristics, as can be seen in Figure 8(b), and relatively low energy dissipative potentials as shown in Figure 8(c).

From the above comparison, it can be deduced that the wave attenuation performance of the SCB model may be as good as other breakwaters. It would potentially reduce the navigational risk in the vicinity of the breakwater because it is a poor wave reflector and a good energy dissipater.

(a) Wave Transmission

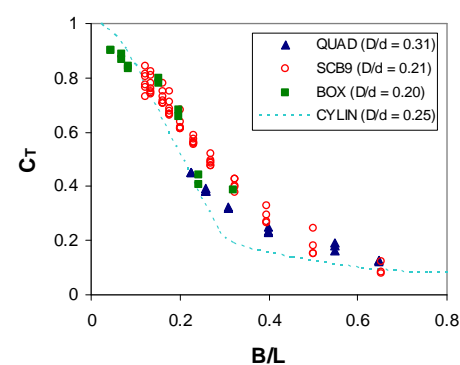

(b) Wave Reflection

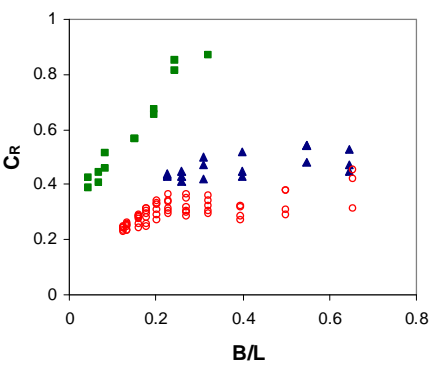

(c) Wave Dissipation

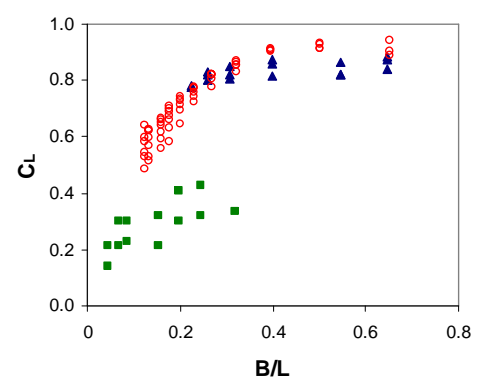

Figure 8. Comparison of energy coefficients [QUAD (Sundar \& Subbarao 2003); SCB9 (the present test model); BOX (Koutandos \& Prinos 2005); CYLIN (Li et al. 2005)]

\section{Predictive Equations}

An attempt is made to develop the empirical formulae for the prediction of the overall hydraulic performance of the SCB with regard to $B / L_{p}$ and $D / d$. As mentioned earlier, wave steepness is a poor governing parameter to $C_{T}, C_{R}$ and $C_{L}$; therefore, it is excluded from the parametric analysis. Considering the area of the fluid domain under a complete cycle of an incident wave $(d \times L)$ and the area of the fluid domain embodied by the free-surface semicircular breakwater $(D \times B)$, the ratio of which can be denoted by $\psi_{a}=D B / d L_{p}$. The degree of wave interception by the structure is directly proportional to $\psi_{a}$.

Figure 9 shows the relationship of $C_{T}, C_{R}$ and $C_{L}$ with respect to $\psi_{a}$ in three tested $D / d$ cases. A distinct relationship is developed between $C_{T}$ and $\psi_{a}$ as can be seen in Figure 9(a). In Figure 9(b), the $C_{R}$ values almost linearly increase with the increase in $\Psi_{a}$. As $\Psi_{a}>0.05$, the influence of $D / d$ begins to dominate over $C_{R}$; thereby giving different sets of predictive equation for $C_{R}$ for respective $D / d$. As for 
$C_{L}$, a positive relationship is observed at $\Psi_{a}<0.07$, and the $C_{L}$ values remain above 0.85 thereafter as shown in Figure 9(c). The empirical formulae for the prediction of $C_{T}$ and $C_{R}$ for the SCB model are summarized in Table 1 . The energy loss coefficient $C_{L}$ can be directly computed by Equation (3). It is important to emphasis that the Equations (6) to (9) are valid only when the following test limits are fulfilled:

$$
\frac{B}{d} \approx 0.7 ; \quad 0.10<\frac{B}{L_{p}}<0.70 ; \quad 0.01<\frac{H_{m 0}}{L_{p}}<0.10 \text { and } 0.07<\frac{D}{d}<0.22
$$

The predicted results obtained from the empirical model are validated against the experimental results in Figure 10. It is evident that the empirical equations proposed in Table 1 are capable of predicting the $C_{T}, C_{R}$ and $C_{L}$ of the SCB model with error bands of $\pm 10 \%, \pm 20 \%$ and $\pm 20 \%$, respectively. These equations are simple and particularly useful to provide quick estimation of the overall performance of the SCB.
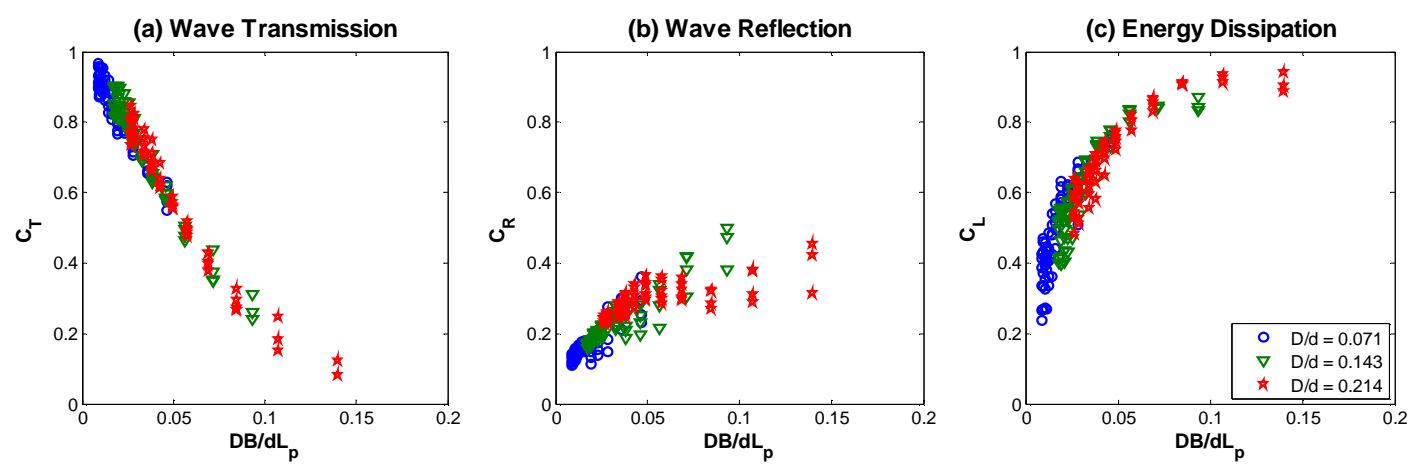

Figure 9. $C_{T}, C_{R}$ and $C_{L}$ of the SCB model with respect to $\Psi_{a}$.

Table 1. Predictive equations for $C_{T}$ and $C_{R}$.

\begin{tabular}{|c|c|c|c|}
\hline Coefficients & Range of $\Psi_{a}$ & Empirical Equations & \\
\hline Transmission & $\Psi_{a}<0.15$ & $C_{T}=27.4 \Psi_{a}^{2}-10.8 \Psi_{a}+1.03$ & (6) \\
\hline \multirow[t]{3}{*}{ Reflection } & $\Psi_{a}<0.05$ & $C_{R}=-34.2 \Psi_{a}^{2}+6.48 \Psi_{a}+0.071$ & (7) \\
\hline & $0.05<\Psi_{a}<0.10$ & $D / d=0.143: \quad C_{R}=-66.8 \Psi_{a}^{2}+14.7 \Psi_{a}-0.302$ & (8) \\
\hline & $0.05<\Psi_{a}<0.15$ & $D / d=0.214: \quad C_{R}=34.5 \Psi_{a}{ }^{2}-5.45 \Psi_{a}+0.524$ & (9) \\
\hline
\end{tabular}

(a) Wave Transmission

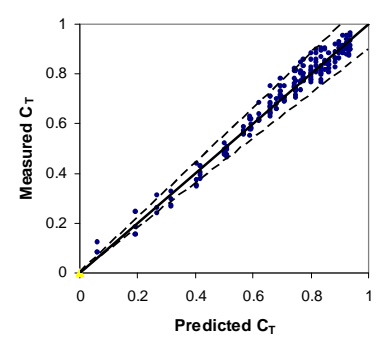

$\mathrm{R}^{2}=0.97$

Discrepancy: $\pm 10 \%$ (b) Wave Reflection

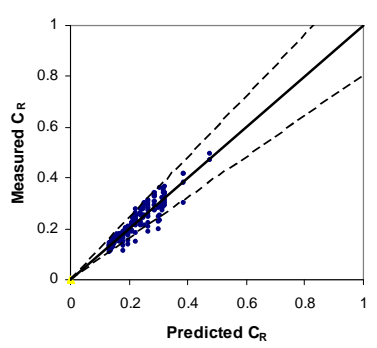

$\mathrm{R}^{2}=0.83$

Discrepancy: $\pm 20 \%$ (c) Wave Dissipation

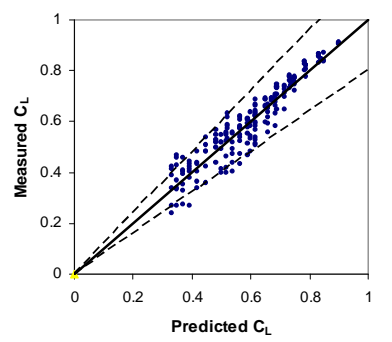

$\mathrm{R}^{2}=0.90$

Discrepancy: $\pm 20 \%$

Figure 10. Predicted results validated against experimental results. 


\section{Wave Climate at the Breakwater}

Figure 11 presents the wave climate at the breakwater in the form of wave modification coefficients, i.e. $C_{F}$ and $C_{C}$, plotted against $B / L_{p}$ for three depths of immersion. Figure 11(a) demonstrates the presence of the SCB model causes an amplification of $C_{F}$ to a maximum value of 1.4. The increased wave activity in front of the model is mainly attributed to (i) abrupt flow restriction due to the front wall of the SCB; and (ii) interaction between the incident and reflected waves near the front wall. A mild increase of $C_{F}$ with the increasing $B / L_{p}$ is observed for $D / d=0.071$ and 0.143 . However, the $C_{F}$ of $D / d=0.214$ has the reverse trend. This may be due to the decrease of wave reflection at higher range of $B / L_{p}$ as can be seen in Figure $4(b)$.

Figure 11(b) shows that wave agitation within the breakwater chamber was rather more controlled compared to that occurred in front of the breakwater. A maximum $C_{C}$ of 1.2 is recorded for $B / L_{p}<0.4$ regardless of the variation of $D / d$. As $B / L_{p}$ increases beyond 0.4 , wave calming effect (i.e. $\left.H_{m 0, c}<H_{m 0, i}\right)$ is observed in the breakwater's chamber.
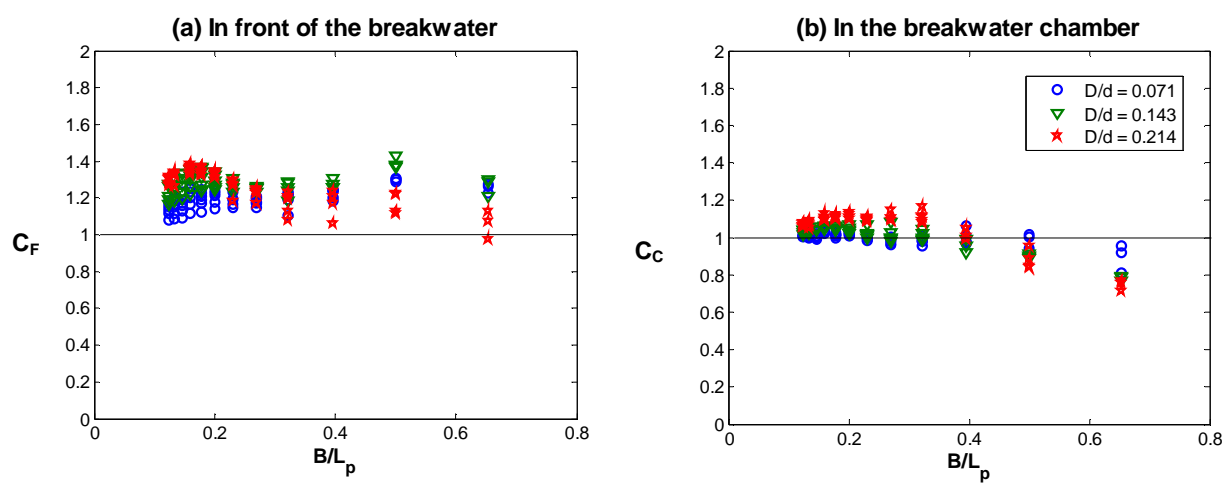

Figure 11. Wave climate at the front and in the SCB model.

\section{Horizontal Wave Force}

Figure 12 presents the average of the highest one-third horizontal loads due to wave crest, $F_{1 / 3}$ acting on the SCB model, against the significant wave period, $T_{s}$ and the significant wave height $H_{s}$. The plots for respective $D / d$ in Figure 12(a) show no explicit relation between $F_{1 / 3}$ and $T_{p}$. On the other hand, $F_{1 / 3}$ is strongly related with $H_{s}$ in all the tested $D / d$ in Figure 12(b). Therefore, the effect of the wave period and the corresponding wave length are excluded from the analysis of horizontal wave forces. The horizontal force due to wave crest is normalised by $F_{1 / 3} / \rho g B b H_{s}$, where $\rho=$ the density of the fluid, $g=$ the acceleration due to gravity, and $b=$ the length of the SCB perpendicular to wave direction.

In Figure 13(a), the wave force coefficient is plotted against the wave height-to-breakwater draft ratio, $H_{S} / D$ in three depths of immersion. The exposure area of the SCB to the wave attack is anticipated to be directly proportional to the increase of $H_{s} / D$. It is seen from this plot that the force coefficient for each $D / d$ increases almost linearly with the increase in $H_{s} / D$. As expected, the breakwater with larger exposure area to wave attack will receive greater horizontal wave impact, and vice versa. The larger the breakwater draft, the greater will be the horizontal wave force acting on the SCB model. The ratios between the maximum horizontal wave force, $F_{\max }$ and the corresponding $F_{1 / 3}$ are shown in Figure 13(b). A best-fit linear regression line is plotted to characterise the behaviour of $F_{\max } / F_{1 / 3}$ with respect to $H_{s} / D$ for all cases of $D / d$. In general, $F_{\max }$ is approximately $1.5-2.3,1.6-2.4$ and $1.6-3.0$ times higher than $F_{1 / 3}$ for $D / d=0.071,0.143$ and 0.214 , respectively, within the test limits of the experiments. 
(a) The effect of $T_{s}$

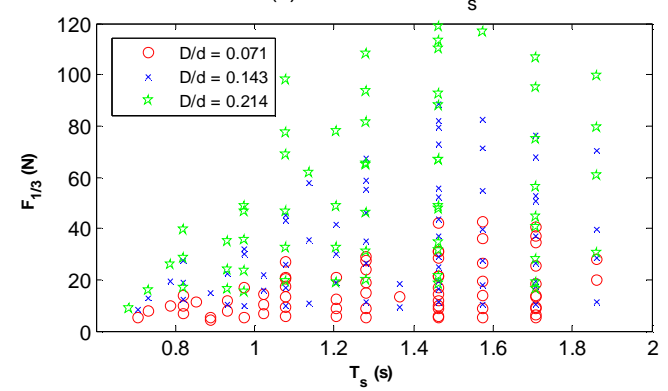

(b) The effect of $\mathrm{H}_{\mathrm{s}}$

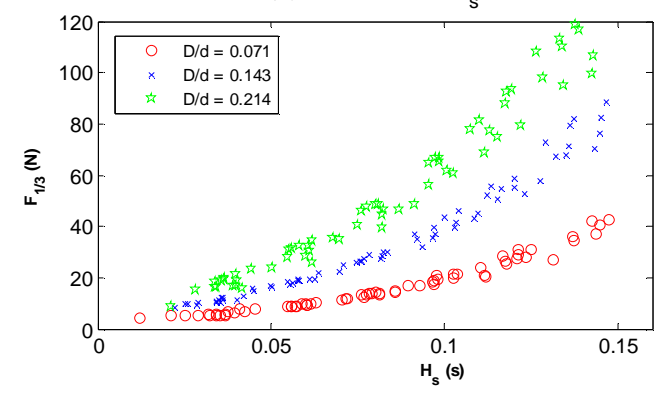

Figure 12. The average highest one-third horizontal wave force, $F_{1 / 3}$ verses (a) the significant wave period, $T_{s}$ and (b) the significant wave height, $H_{s}$.

(a)

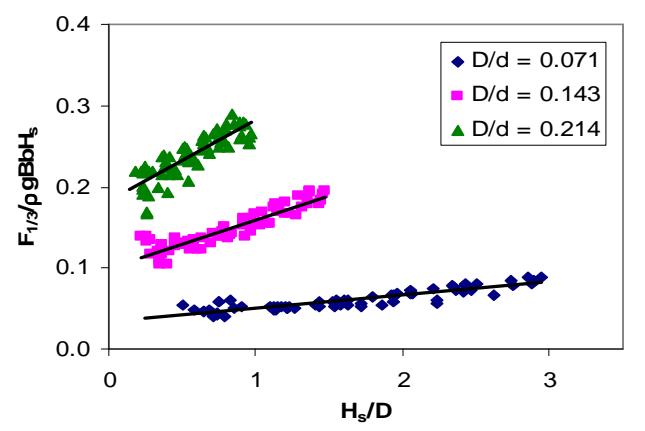

(b)

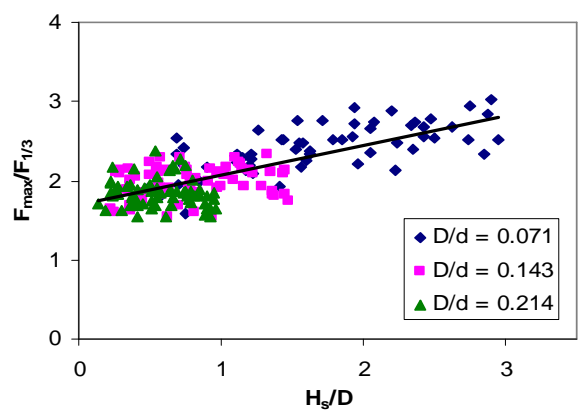

Figure 13. Dimensionless horizontal wave forces on the SCB model - (a) $F_{1 / 3} / \rho g B b H_{s}$, and (b) $F_{\max } / F_{1 / 3}$

\section{CONCLUSIONS}

Factors influencing the perforated free surface SCB were investigated by physical model tests. The energy coefficients $C_{T}, C_{R}$ and $C_{L}$ are strongly governed by the relative width and draft of the breakwater; however, they are less affected by the wave steepness. Wave attenuation and dissipative characteristics of the SCB improve as the relative width and (or) draft of the breakwater increases. The breakwater has low reflective characteristics with the overall reflected energy of less than $25 \%$ of the incident wave energy within the test limit. On the other hand, the structure becomes highly dissipative (more than 50\% in energy level) when immersed to greater drafts. The perforated SCB is indeed a good energy dissipater with low wave reflection and its wave attenuation is almost comparable to some of the typical free surface breakwaters. Therefore, the performance characteristics exhibited by the SCB may be favourable to the design of the marine infrastructures.

Parametric analysis of the experimental data provided design formulae for wave transmission and reflection as a function of $\psi_{a}\left(=D B / d L_{p}\right)$. The computed results are validated against the experimental data yielding reasonably good correlations. Hence, the proposed formulae could serve as handy 'tool' for quick estimation of the hydraulic performance of the perforated SCB within the test limits.

The wave excitation in front of the breakwater is more significant than that in the breakwater's chamber. The maximum wave height measured in front of the breakwater is approximately $20 \%$ higher than that measured in the breakwater's chamber. The wave condition in the chamber is relatively calm when subjected to smaller period waves.

The horizontal wave force on the SCB model is strongly dependent on the wave height and immersion depth rather than the wave period. The maximum horizontal wave forces are higher than the average of the largest one-third horizontal wave force by a factor ranging from 1.5 to 3 depending upon the $D / d$ and $H_{s} / D$. 


\section{ACKNOWLEDGEMENT}

This research work was supported by the Institute for Energy Systems of School of Engineering at the University of Edinburgh. The first author's studentship was funded by Universiti Teknologi PETRONAS, Malaysia. The authors wish to express their gratitude to Professor William Allsop from HR Wallingford for his valuable comments.

\section{REFERENCES}

Brossard, J., Jarno-Druaux, A., Marin, F. and Tabet-Aoul, E.H. 2003. Fixed absorbing semi-immersed breakwater. Coastal Engineering, 49, 25-41.

Günaydın, K. and Kebdaşl1, M.S. 2004. Performance of solid and perforated U-type breakwaters under regular and irregular waves, Ocean Engineering, 31, 1377-1405.

Günaydın, K. and Kebdaşlı, M.S. 2007. Investigation of П-type breakwater performance under regular and irregular waves. Ocean Engineering, 34, 1028-1043.

Hsiao, S-,S., Fang. H-,M., Chang, C-,M. and Lee, T-,S. 2008. Experimental study of the wave energy dissipation due to the porous-piled structure. Proceedings of the $18^{\text {th }}$ International Offshore and Polar Engineering Conference, Vancouver, Canada, 592-598.

Hu, H., Wang, K.H. and Williams, A.N. 2002. Wave motion over a breakwater system of a horizontal plate and a vertical porous wall. Ocean Engineering, 29, 373-386.

Hughes, S.A. 1993. Physical models and laboratory techniques in coastal engineering. World Scientific, Singapore.

Koftis, T. and Prinos, P. 2005. On the hydrodynamic efficiency of floating breakwaters. Arabian Coast Conference.

Koutandos, E.V. 2007. Hydrodynamic analysis of a skirt breakwater, Maritime Engineering, 160, Institute of Civil Engineers, 121-133.

Koutandos, E. and Prinos, P. 2005. Design formulae for wave transmission behind floating breakwaters, IAHR XXXI Congress, Seoul, Korea, 4081-4089.

Li, D., Panchang, V., Tang, Z., Demirbilek, Z., and Ramsden, J. 2005. Evaluation of an approximate method for incorporating floating docks in harbor wave prediction models, Canadian Journal of Civil Engineering, 32, 1082-1092.

Mansard, E.P.D. and Funke, E.R. 1980. The measurement of incident and reflected spectra using a least squares method, Proceedings of the $17^{\text {th }}$ International Conference on Coastal Engineering, ASCE, 154-172.

Neelamani, S. and Gayathri 2006. Wave interaction with twin plate wave barrier. Ocean Engineering, 33, 495-516.

Neelamani, S. and Rajendran, R. 2002a. Wave interaction with T-type breakwaters. Ocean Engineering, 29, 151-175.

Neelamani, S. and Rajendran, R. 2002b. Wave interaction with ' $\perp$ '-type breakwaters. Ocean Engineering, 29, 561-589.

Neelamani, S. and Vedagiri, M. 2002. Wave interaction with partially immersed twin vertical barriers. Ocean Engineering, 29, 215-238.

Patarapanich, M and Cheong, H. F. 1989. Reflection and transmission characteristics of regular and random waves from a submerged horizontal plate. Coastal Engineering, 13, 161-182.

Sundar, V. and Subba Rao, B.V.V. 2003. Hydrodynamic performance characteristics of quadrant frontface pile-supported breakwater. Journal of Waterway, Port, Coastal and Ocean Engineering, ASCE, 22-33.

Tsay, T.-K. and Liu, P.L.-F. 1983. A finite element model for wave refraction and diffraction. Applied Ocean Research, 5(1), 30-37.

Usha, R. and Gayathri, T. 2005. Wave motion over a twin-plate breakwater. Ocean Engineering, 32 , 1054-1072.

Wang, Y., Wang, G. and Li, G. 2006. Experimental study on the performance of the multiple-layer breakwater. Ocean Engineering, 33, 1829-1839. 